Pacific Journal of Mathematics

APPROXIMATION TO BOUNDED HOLOMORPHIC
FUNCTIONS ON STRICTLY PSEUDOCONVEX DOMAINS 


\title{
APPROXIMATION TO BOUNDED HOLOMORPHIC FUNCTIONS ON STRICTLY PSEUDOCONVEX DOMAINS
}

\author{
R. Michael Range
}

\begin{abstract}
This paper investigates the problem of approximating bounded holomorphic functions on a strictly pseudoconvex domain $D$ by functions in $H^{\infty}(D)$ which extend analytically across a given subset $E$ of the boundary of $D$. In particular, it is shown that if $f \in H^{\infty}(D)$ extends continuously to $E$, one obtains uniform approximation on $D$.
\end{abstract}

A recent result of I. Lieb [7] and N. Kerzman [6] states that any continuous function on the closure of a strictly pseudoconvex domain $D$ with smooth boundary which is holomorphic on $D$, can be approximated uniformly on $\bar{D}$ by functions holomorphic in a neighborhood of $\bar{D} .^{1}$ Here we prove the following theorem, which contains the above mentioned result in case $E=\partial \partial . \quad H^{\infty}(D)$ denotes the Banach algebra of bounded holomorphic functions on $D$.

Theorem 1. Let $D$ be a bounded, strictly pseudoconvex domain in $C^{n}$ with $C^{4}$ boundary, and let $E$ be any subset of the boundary $\partial D$ of $D$. Then every bounded holomorphic function on $D$ which extends continuously to $E$ can be approximated uniformly on $D$ by functions in $H^{\infty}(D)$ which extend analytically across $E$.

Part of our proof uses the argument of Lieb and Kerzman, with some additional estimates, so we do not obtain a new proof when $E=\partial D$. In case $D$ is the unit disc in the complex plane, Theorem 1 was proved by T. W. Gamelin and J. Garnett [3]. ${ }^{2}$ We show in $\S 5$ that the ideas of our proof, when applied to this special case, simplify their proof considerably.

We also obtain the following theorem, which, for $E=\partial D$, contains the bounded pointwise approximation theorem noted by N. Kerzman [6].

Theorem 2. Let $D$ and $E$ be as in Theorem 1. To every $f \in$ $H^{\infty}(D)$ there exists a sequence of functions $F_{m} \in H^{\infty}(D), m=1,2, \cdots$, which have the following properties:

1 This theorem has been proved also by G. M. Henkin, Integral Representations of Functions Holomorphic in Strictly Pseudoconvex Domains and some Applications, Math. USSR Sbornik, 7 (1969), 597-616.

${ }^{2}$ For open subsets $E$ of the boundary of the unit disc this result was obtained first by A. Stray, An Approximation Theorem for Subalgebras of $\mathrm{H}^{\infty}$, Pacific J. Math., 35 (1970), 511-515. 
(i) $\left\|F_{m}\right\|_{\infty} \leqq C\|f\|_{\infty}, m=1,2, \cdots$

(ii) Each $F_{m}$ extends analytically across $E$.

(iii) $F_{m}$ converges to $f$ uniformly on any subset of $D$ whose closure is disjoint from $\bar{E}$ (or only disjoint from $E$, in case $E$ is a $G_{\delta}$ ).

Theorems 1 and 2 will follow quite easily from:

Theorem 3. Let $D$ and $E$ be as in Theorem 1 , and let $f \in H^{\infty}(D)$. Assume that there is an open neighborhood $W$ of $E$ and a continuous function $u: W \rightarrow C$, such that for some $d>0$

$$
|f(z)-u(z)|<d \text { for } z \in D \cap W .
$$

Let $S$ be any subset of $D$ such that $\bar{S} \cap E=\varnothing$ and let $\varepsilon>0$ be given.

One can then find $F \in H^{\circ}(D)$ such that

(1) $F$ extends analytically across $E$.

(2) $|F(z)-f(z)|<\varepsilon$ for $z \in S$.

(3) $|F(z)-f(z)|<C_{1} d$ for all $z \in D$.

REMARK. The proof given here shows that the constants $C$ and $C_{1}$ depend only on the domain $D$, and that they can be chosen independently of small $C^{4}$ perturbations of $\partial D$.

2. Preparations. If $z \in C^{n}$, we set $z=\left(z_{1}, \cdots, z_{n}\right)$ with $z_{j}=x_{2 j-1}+$ $i x_{2 j}, j=1, \cdots, n$, where $x_{1}, \cdots, x_{2 n}$ are the underlying real coordinates of $\boldsymbol{C}^{n}$. The norm of $z$ is given by $\|z\|=\left(\sum_{j=1}^{n} z_{j} \bar{z}_{j}\right)^{1 / 2}$. For a multiindex $\gamma=\left(\gamma_{1}, \cdots, \gamma_{2 n}\right), \gamma_{j}$ nonnegative integers, we set $|\gamma|=\sum_{j=1}^{2 n} \gamma_{j}$ and

$$
D^{\prime}=\frac{\hat{o}^{|r|}}{\partial x_{1}^{\gamma}+\cdots \cdots \partial x_{2 n}^{r_{2 n}}}
$$

Recall that the complex differential operators are defined by

$$
\frac{\partial}{\partial z_{j}}=\frac{1}{2}\left(\frac{\partial}{\partial x_{2 j-1}}+\frac{1}{i} \frac{\partial}{\partial x_{2 j}}\right), \frac{\partial}{\partial \bar{z}_{j}}=\frac{1}{2}\left(\frac{\partial}{\partial x_{2 j-1}}-\frac{1}{i} \frac{\partial}{\partial x_{2 j}}\right),
$$

and that $\bar{\partial} u=\sum_{j=1}^{n}\left(\partial u / \partial \bar{z}_{j}\right) d \bar{z}_{j}$. The function $u$ is holomorphic if and only if $\bar{\partial} u=0$.

For a $C^{4}$ function $\phi$ defined on an open set $U \subset C^{n}$ we set

$$
\|\phi\|_{k}=\sum_{|\gamma| \leqq k} \sup _{z \in U}\left|D^{\gamma} \phi(z)\right|, k=1, \cdots, 4 \text {. }
$$

Let $D \subset \subset C^{n}$ be a strictly pseudoconvex domain with $C^{4}$ boundary. This is equivalent to the following (see Gunning and Rossi [5], chapter IX): there is an open neighborhood $U$ of $\partial D$, a $C^{4}$ function $\rho: U \rightarrow \boldsymbol{R}$ and positive constants $A, B$ such that

(a) $D=\{z \in U: \rho(z)<0\} \cup(D-U)$.

(b) $\quad \sum_{i, j}\left(\partial^{2} \rho(z)\right) /\left(\partial z_{i} \partial \bar{z}_{j}\right) w_{i} \bar{w}_{j} \geqq A\|w\|^{2}, z \in U, w \in C^{n}$, i.e., $\rho$ is strictly 
plurisubharmonic.

(c) $\|\operatorname{grad} \rho(z)\| \geqq B, z \in U$.

In the following we will call such a domain a SSPC domain (smooth, strictly pseudoconvex domain).

It is well known that a small $C^{4}$ perturbation of a SSPC domain gives again a SSPC domain. More precisely, if $\hat{\rho} \in C_{0}^{4}(U)$ and $\|\hat{\rho}\|_{2} \leqq$ $\min \left(A / 2 n^{2}, B / 2\right)$, then $\hat{D}=\{z \in U:(\rho-\hat{\rho})(z)<0\} \cup(D-U)$ is also a SSPC domain.

We will need the following sharper version due to Kerzman [6] of a theorem of Grauert-Lieb [4].

Theorem 4. Let $D$ be a SSPC domain. There is a constant $K>0$ such that for each bounded $C_{(0,1)}^{\infty}-$ form $\alpha=\sum_{\jmath=1}^{n} \alpha_{j} d \bar{z}_{j}$ on $D$ with $\bar{\partial} \alpha=$ 0 , there is a continuous function $u: \bar{D} \rightarrow C, u \in C^{\infty}(D)$, which satisfies $\bar{\partial} u=\alpha$ on $D$ and

$$
\|u\|_{L_{(D)}^{p}} \leqq K\|\alpha\|_{K_{(D)}^{p}}, 1 \leqq p \leqq \infty .
$$

Here $\|\alpha\|_{L^{p}}=\sum_{j=1}^{n}\left\|\alpha_{j}\right\|_{L^{p}}$. Note that $u$ is continuous up to the boundary of $D$, even though $\alpha$ may only be defined on $D$. The constant $K$ can be chosen independently of small $C^{4}$ perturbations of $D$, i.e., the same $K$ will work for the domains $\hat{D}$ defined above, provided $\|\hat{\rho}\|_{2}$ is sufficiently small.

3. The main Lemma. The basic step in the proof of Theorem 3 will be to prove it for the case of closed subsets of $\partial D$. We formulate this part as a separate lemma.

Lemma 1. Let $D$ be a SSPC domain, and assume that $E \subset \partial D$ is closed, while $f, W, u$ and $d$ are as in Theorem 3. Let $S \subset D, \bar{S} \cap E=$ $\varnothing$, and let $\varepsilon>0$ be given. We can then find $F \in H^{\infty}(D)$ such that

(1') $F$ extends analytically across $E$.

$\left(2^{\prime}\right) \quad|F(z)-f(z)|<\varepsilon, z \in S$.

(3') $|F(z)-f(z)|<C_{2} d, z \in D$.

The constant $C_{2}$ depends only on $D$, and it can be chosen independently of small $C^{4}$ perturbations of $D$.

Proof. By shrinking $W$ we can assume that $\bar{W} \cap \bar{S}=\varnothing$. Choose $\chi \in C_{0}^{\infty}(W), 0 \leqq \chi \leqq 1, \chi=1$ on an open neighborhood of $E$. Following our remarks in $\S 2$, if we choose $\hat{\rho} \in C_{0}^{\infty}(U)$ with $\|\hat{\rho}\|_{2}$ small, $\hat{\rho}=0$ on supp $\bar{\partial} \chi, \hat{\rho}>0$ on $E \cup(\partial D-W)$, and otherwise $\hat{\rho} \geqq 0$, we can construct a SSPC domain $\hat{D}$, such that

(4) $D \cup E \subset \hat{D}, \partial D-W \subset \hat{D}$, hence $\mathrm{S} \subset \subset \hat{D}$.

(5) $D_{1}=D \cup(\hat{D}-\operatorname{supp} \chi)$ is a SSPC domain.

(6) $\hat{D} \cap \operatorname{supp} \bar{\partial} \chi \subset D$.

It follows from (6) that 


$$
\alpha= \begin{cases}\bar{\partial}(\chi f) & \text { on } D \\ 0 & \text { on } \hat{D}-D\end{cases}
$$

is a well-defined bounded $C_{(0,1)}^{\infty}-$ form on $\hat{D}$ with $\bar{\partial} \alpha=0$. By Theorem 4 there is a function $p \in C^{\infty}(\hat{D}) \cap C(\overline{\hat{D}})$ with $\bar{\partial} p=\alpha$. Hence the function

$$
h=\left\{\begin{array}{l}
\chi f-p \text { on } D \\
-p \text { on } \hat{D}-\operatorname{supp} \chi \subset D_{1}
\end{array}\right.
$$

is in $H^{\circ}\left(D_{1}\right)$, and its restriction to $D$ is in $H^{\infty}(D)$. Since $\chi=1$, and hence $\bar{\partial} p=0$, in an open neighborhood of $E$, we also obtain that

(8) $f-h$ extends analytically across $E$.

Now $f-h=p$ on $S$, and $p$ may not be small there. Therefore we want to approximate $h$ by functions holomorphic in a neighborhood of $\bar{D}_{1}$. Kerzman [6] showed that there is a bounded sequence $\left\{h_{k}\right\}_{k=1}^{\infty}$ of functions holomorphic on $\bar{D}_{1}$ which converges to $h$ uniformly on compact subsets of $D_{1}$. By (4) and (5), $S \subset \subset D_{1}$, thus we can choose $k_{0}$ so large that $\left|h(z)-h_{k_{0}}(z)\right|<\varepsilon$ for $z \in S$. The function

$$
F=f-h+h_{k_{0}}
$$

clearly satisfies $\left(2^{\prime}\right)$, and from (8) we also obtain $\left(1^{\prime}\right)$. Thus we only have to prove $\left(3^{\prime}\right)$, which requires an estimate on all of $D$ which we cannot deduce immediately from Kerzman's result. For the sake of completeness we will repeat the proof in [6], adding the estimates needed in our case.

As in [6], we choose a covering of $\partial D$ by balls $B_{i}$ with center $p_{i} \in \partial D, i=1, \cdots, r$, and we let $U_{i}=D_{1} \cap B_{i}$. Denote by $n_{i}$ the unit outer normal to $\partial D$ at $p_{i}$. For fixed $\delta>0$ let $U_{i}^{\delta}=U_{i}+\delta n_{i}, i=1$, $\cdots, r$; we also set $U_{0}^{o}=U_{0}=D_{1}$. The covering $\left\{B_{i}\right\}$ can be chosen in such a way that $D_{1} \subset \subset \bigcup_{i=0}^{r=0} U_{i}^{o}$ for all sufficiently small $\delta>0$. For each such $\delta$ we then can find a SSPC domain $D_{1}^{o}$ satisfying

$$
D_{1} \subset \subset D_{1}^{\delta} \subset \subset \bigcup_{i=0}^{r} U_{i}^{\delta} \text {. }
$$

If we denote by $\mathscr{F}\left(U_{i}\right)$ the set of complex valued functions on $U_{i}$, we can define the translation operators

$$
T_{i}^{j}: \mathscr{F}\left(U_{i}\right) \rightarrow \mathscr{F}\left(U_{i}^{\hat{o}}\right)
$$

in the following way:

For $i=0, T_{0}^{o}$ is the identity operator, and for $i=1, \cdots, r,\left(T_{i}^{\delta} \psi\right)(z)=$ $\psi\left(z-\delta n_{i}\right)$. In particular we set $v_{i}^{o}=T_{i}^{o} h, i=0, \cdots, r$, where $h$ is the function defined in (7). Let $v_{i}^{\delta}$ also denote its restriction to $V_{i}^{\delta}=$ $U_{i}^{\delta} \cap D_{1}^{\delta}$.

The functions $v_{i}^{\delta}$ define a holomorphic cocycle $v_{i j}^{\delta}=v_{i}^{\delta}-v_{j}^{\delta}$ subordinate to the covering $\left\{V_{i}^{i}\right\}_{i=0}^{r}$ of $D_{1}^{\delta}$. Using Theorem 4 one finds 
holomorphic functions $h_{i}^{\delta}: V_{i}^{\delta} \rightarrow C$ such that $h_{i}^{\delta}-h_{j}^{\delta}=v_{i j}^{\delta}$ on $V_{i}^{\delta} \cap V_{j}^{\delta}$ and (9)

$$
\left\|h_{i}^{\hat{\delta}}\right\|_{L^{p}\left(V_{i}^{\delta}\right)} \leqq \widetilde{K} \max _{0 \leqq k, j \leqq r}\left\|v_{k j}^{\delta}\right\|_{L^{p}\left(V_{k}^{\delta} \cap \cap V_{j}^{\delta}\right)}
$$

for $1 \leqq p \leqq \infty . ~ \widetilde{K}$ depends only on the constant $K$ of Theorem 4 and on the covering $\left\{B_{i}\right\}$ of $\partial D$, hence it is independent of small $C^{4}$ perturbations of $D$.

For $p \geqq 1$ but $p \neq \infty, v_{i}^{o}=T_{i}^{\delta} h$ converges to $h$ in $L^{p}$-norm for $\delta \rightarrow 0$, hence $\left\|v_{i j}^{\hat{i}}\right\|_{L^{p}\left(V_{i}^{\tilde{\delta}} \cap V_{j}^{\delta}\right)} \rightarrow 0$, and by (9) also

$$
\left\|h_{i}^{\delta}\right\|_{L^{p}\left(V_{i}^{\delta}\right)} \rightarrow 0,1 \leqq p<\infty,
$$

for $\delta \rightarrow 0$.

Thus the holomorphic functions $h^{\delta}: D_{1}^{\delta} \rightarrow C$ defined by

$$
h^{o}(z)=v_{i}^{\delta}(z)-h_{i}^{\delta}(z) \text { for } z \in V_{i}^{o}
$$

converge to $h$ in $L^{p}\left(D_{1}\right)$, and we also obtain uniform convergence on compact subsets of $D_{1}$. Hence, for $\delta$ sufficiently small, we can set $h_{k_{0}}=h^{\delta}$ to obtain $\left|h_{k_{0}}(z)-h(z)\right|<\varepsilon$ for $z \in S$. We now prove

$$
\left|h_{k_{0}}(z)-h(z)\right|<C_{2} d \text { for all } z \in D_{1},
$$

which will imply $\left(3^{\prime}\right)$.

To obtain (10) we will first show the estimate

$$
\sup _{z \in V_{i}^{\hat{o}} \cap v_{j}^{\hat{o}}}\left|v_{i j}^{\delta}(z)\right|<4 d, \quad i, j=0, \cdots, r
$$

for small $\delta$. Note that $v_{i j}^{\delta}=T_{t}^{\delta} h-T_{j}^{o} h$, and hence

$$
v_{i j}^{o}=T_{i}^{j}(\chi f)-T_{j}^{\delta}(\chi f)-\left(T_{i}^{\delta} p-T_{j}^{i} p\right) \text {. }
$$

Extend $\chi u$ to be zero outside of $W$. Since $\chi u$ and $p$ are uniformly continuous on $\bar{D}_{1}$, for sufficiently small $\delta$ we will have

$$
\left|\left(T_{i}^{\delta} p\right)(z)-\left(T_{j}^{\delta} p\right)(z)\right|<d, \quad\left|T_{i}^{\delta}(\chi u)(z)-T_{j}^{\delta}(\chi u)(z)\right|<d
$$

for $z \in V_{i}^{i} \cap V_{j}^{\delta}$. Also it follows from $(*)$ that $|(\chi f)(z)-(\chi u)(z)|<d$ for $z \in D_{1}$.

Hence for $z \in V_{i}^{\delta} \cap V_{j}^{\delta}$

$$
\left|T_{i}^{\hat{o}}(\chi f)(z)-T_{j}^{\hat{\delta}}(\chi f)(z)\right|
$$

$$
\leqq\left|T_{i}^{\delta}(\chi f-\chi u)(z)\right|+\left|T_{i}^{\delta}(\chi u)(z)-T_{j}^{\delta}(\chi u)(z)\right|+\left|T_{j}^{o}(\chi u-\chi f)(z)\right|<3 d .
$$

(11) now follows from (12), (13) and (14).

From (9) and (11) we now get

$$
\sup _{z \in V_{i}^{\delta}}\left|h_{i}^{\delta}(z)\right|<\widetilde{K} 4 d .
$$


Let $z \in V_{i}^{\delta} \cap D_{1}$. Then $\left|h^{\delta}(z)-h(z)\right|=\left|v_{i}^{\delta}(z)-h_{i}^{\delta}(z)-h(z)\right| \leqq \mid v_{i}^{\delta}(z)-$ $h(z)|+| h_{i}^{\delta}(z) \mid$. Noting that $h=T_{0}^{\delta} h=v_{0}^{\delta}$, (11) shows that $\mid v_{i}^{\delta}(z)-$ $h(z) \mid<4 d$. Together with (15) we thus obtain the desired estimate if we set $C_{2}=4+4 \widetilde{K}$.

4. Proofs of the Theorems. To prove Theorem 3, we have to extend Lemma 1 to arbitrary subsets of the boundary. The recursive argument we use is similar to the one in [3], although in our case we also have to modify the domain at each step.

Let $E \subset \partial D$ be arbitrary now. We can again assume that $\bar{S} \cap$ $W=\varnothing$. Moreover, replacing $E$ by $W \cap \partial D$ we can also assume that $E$ is open in $\partial D$. It is easy to construct two families $\left\{Q_{i}\right\}_{i=1}^{\infty},\left\{\widetilde{Q}_{i}\right\}_{i=1}^{\infty}$ of closed subsets of $\partial D$, and open neighborhoods $V_{i}$ (resp. $\left.\widetilde{V}_{i}\right)$ of $Q_{i}($ resp. $\left.\widetilde{Q}_{i}\right)$ such that

$$
E=\left(\bigcup_{i=1}^{\infty} Q_{i}\right) \cup\left(\bigcup_{i=1}^{\infty} \widetilde{Q}_{i}\right)
$$

and

$$
V_{i} \cap V_{j}=\varnothing, \widetilde{V}_{i} \cap \widetilde{V}_{j}=\varnothing \text { for } i \neq j .
$$

Hence also the $Q_{i}$ respectively the $\widetilde{Q}_{i}$ are pairwise disjoint. We clearly may also assume that $V_{i}, \widetilde{V}_{i} \subset W$, hence $V_{i} \cap \bar{S}=\varnothing$ and $\tilde{V}_{i} \cap \bar{S}=\varnothing$. Also, let $\varepsilon<d$.

We now construct inductively a sequence of SSPC domains $D_{i}$ and functions $\phi_{i} \in H^{\infty}\left(D_{i}\right)$ such that

$$
\begin{gathered}
D \cup\left(\bigcup_{j=1}^{i} Q_{j}\right) \subset D_{i} \text { and } D_{i}-\left(\bigcup_{j=1}^{i} V_{j}\right) \subset D \\
\left|\phi_{i}(z)-\phi_{i-1}(z)\right|<\varepsilon / 2^{i} \text { for } z \in D_{i-1}-V_{i}, i=1,2, \cdots \\
\left|\phi_{i}(z)-\phi_{i-1}(z)\right|<2 C_{2} d \text { for } z \in D, i=1,2, \cdots .
\end{gathered}
$$

We start with $\dot{\phi}_{0}=f$ and $D_{0}=D$. Assume that we already constructed $\phi_{0}, \cdots, \phi_{i-1}$ and $D_{0}, \cdots, D_{i-1}$ satisfying the above conditions. Note that by (17) $V_{i} \cap D_{i-1} \subset D$. Thus for $z \in V_{i} \cap D_{i-1}$

$$
\begin{aligned}
\left|\dot{\phi}_{i-1}(z)-u(z)\right| & \leqq \sum_{j=1}^{i-1}\left|\dot{\phi}_{j}(z)-\dot{\phi}_{j-1}(z)\right|+\left|\dot{\phi}_{0}(z)-u(z)\right| \\
& <\sum_{j=1}^{i=1} \varepsilon / 2^{j}+d<2 d .
\end{aligned}
$$

Hence Lemma 1 applies to $\dot{\phi}_{i-1} \in H^{\infty}\left(D_{i-1}\right)$ with $Q_{i}$ and $V_{i}$ replacing $E$ and $W$. For $S$ we may choose $D_{i-1}-V_{i}$. Thus we obtain from Lemma 1 a function $\phi_{i} \in H^{\infty}\left(D_{i-1}\right)$ which extends analytically across $Q_{i}$ and such that (18) and (19) hold.

By a small $C^{4}$ perturbation of $D_{i-1}$ we now construct a SSPC domain $D_{i}$ such that $\left(D_{i-1} \cup Q_{i}\right) \subset D_{i} \subset\left(D_{i-1} \cup V_{i}\right)$ and $\dot{\phi}_{i}$ extends to be 
in $H^{\circ}\left(D_{i}\right)$ This completes the inductive step. Note that the domains $D_{i}$ can be constructed in such a way that Lemma 1 will hold for $D_{i}$ with the same constant $C_{2}$.

Since each $z \in D$ is contained in at most one set $V_{i}$ and $S \cap V_{i}=$ $\varnothing, i=1,2, \cdots$, it follows from (18) and (19) that $\sum_{\jmath=1}^{\infty}\left(\phi_{j}-\phi_{j-1}\right)+f$ converges uniformly on compact subsets of $D$ to a function $\Phi \in H^{\infty}(D)$ which satisfies

$$
\begin{gathered}
|\Phi(z)-f(z)|=\left|\sum_{j=1}^{\infty}\left(\dot{\phi}_{j}(z)-\phi_{j-1}(z)\right)\right|<\varepsilon+2 C_{2} d \\
<\left(2 C_{2}+1\right) d \quad \text { for } z \in D . \\
|\Phi(z)-f(z)|<\varepsilon \quad \text { for } z \in S .
\end{gathered}
$$

We also obtain from $(*)$ and $(20)|\Phi(z)-u(z)|<\left(2 C_{2}+1\right) d+d=d^{\prime}$ for $z \in W \cap D$. Since $\Phi=\sum_{j=1}^{\infty}\left(\phi_{j}-\phi_{j-1}\right)+f=\sum_{j=i+1}^{\infty}\left(\phi_{j}-\phi_{j-1}\right)+\dot{\phi}_{i}$ for each $i=1,2, \cdots, \Phi$ extends analytically to each $D_{i}$.

ClaIm. There exists a SSPC domain $\hat{D}$ such that $D \cup\left(\bigcup_{i=1}^{\infty} Q_{i}\right) \subset$ $\hat{D} \subset \bigcup_{i=1}^{\infty} D_{i}, \Phi \in H^{\infty}(\hat{D})$ and $|\Phi(z)-u(z)|<d^{\prime}$ for $z \in W \cap \hat{D}$.

Assuming the claim for a moment, we complete the proof of Theorem 3. We perform the same inductive construction which led from $f$ to $\Phi$ with $\Phi$ and the family $\widetilde{Q}_{i} \cap \partial \hat{D}$. We thus obtain $\Psi \in$ $H^{\infty}(\hat{D})$ with

$$
\begin{gathered}
|\Psi(z)-\Phi(z)|<\left(2 C_{2}+1\right) d^{\prime} \text { for } z \in \hat{D} \\
|\Psi(z)-\Phi(z)|<\varepsilon \text { for } z \in S
\end{gathered}
$$

and $\Psi$ extends analytically across each $\widetilde{Q}_{i} \cap \partial \hat{D}$. Then $F=\Psi \mid D$ is the required function. Clearly $F \in H^{\infty}(D)$, and $F$ extends analytically across $E=\left(\bigcup_{i=1}^{\infty} Q_{i}\right) \cup\left(\bigcup_{i=1}^{\infty} \widetilde{Q}_{i}\right)$. The estimates (2) and (3) follow from $(20)-(23)$ with $C_{1}=\left(2 C_{2}+1\right)\left[1+\left(2 C_{2}+2\right)\right]$, and $\varepsilon$ replaced by $2 \varepsilon$.

It remains to prove the claim. Since $\Phi$ is analytic on $Q_{i}, i=1$, $2, \cdots$, we can shrink the neighborhoods $V_{i}$ and assume that $\Phi$ is analytic on $V_{i}$ and that $|\Phi(z)| \leqq 2 \sup _{w \in D}|\Phi(w)|,|\Phi(z)-u(z)|<d^{\prime}$ for $z \in V_{i}$. Choose $\rho_{i} \in C_{0}^{\infty}\left(V_{i}\right), \rho_{i} \geqq 0, \rho_{i}>0$ on $Q_{i}$ and $\left\|\rho_{i}\right\|_{4} \leqq 1 / 2^{i}$ $\min \left(A / 2 n^{2}, B / 2\right)$, where $A$ and $B$ are the positive constants given in $\S 2$. It follows that $\sum_{i=1}^{\infty} \rho_{i}$ converges uniformly to a function $\hat{\rho} \in C_{0}^{4}(U)$ and that $\sum_{i=1}^{\infty} D^{r} \rho_{i}=D^{\gamma} \hat{\rho},|\gamma| \leqq 4$. The domain $\hat{D}=D \cup\{z \in U: \rho(z)-\hat{\rho}(z)<$ $0\}$ is the required one.

This completes the proof of Theorem 3 .

Proof of Theorem 1. Let $f \in H^{\circ}(D)$ extend continuously to $E$. 
For every $m=1,2, \cdots$ we can choose an open neighborhood $W_{m}$ of $E$ such that the diameter of the cluster set of $f$ at $z \in W_{m} \cap \partial D$ is less than $1 / m$. It is now easy to construct a continuous function $u$ on $W_{m}$ which satisfies condition $(*)$ in Theorem 3 , with $d=1 / m$. We thus obtain functions $F_{m} \in H^{\infty}(D)$ which extend analytically across $E$ and satisfy $\sup _{z \in D}\left|F_{m}(z)-f(z)\right|<C_{1} / m, m=1,2, \cdots$.

Proof of Theorem 2. Let $\left\{S_{m}\right\}_{m=1}^{\infty}$ be an increasing sequence of open subsets of $D$ such that $\bar{S}_{m} \cap \bar{E}=\varnothing$ and $\cup \bar{S}_{m}=\bar{D}-\bar{E}\left(\bar{S}_{m} \cap E=\varnothing\right.$ and $\cup \bar{S}_{m}=\bar{D}-E$, in case $E$ is a $\left.G_{\dot{o}}\right)$. The hypotheses of Theorem 3 are satisfied with $S=S_{m}, \varepsilon=1 / m, W$ any neighborhood of $E, u \equiv 0$ and $d=3 \sup _{z \in D}|f(z)|$. Hence Theorem 3 yields functions $F_{m} \in H^{\infty}(D)$, $m=1,2, \cdots$, which clearly satisfy (ii) and (iii). From (3) we also obtain, for $z \in D$,

$$
\left|F_{m}(z)\right| \leqq|f(z)|+\left|F_{m}(z)-f(z)\right| \leqq\left(1+3 C_{1}\right) \sup _{z \in D}|f(z)|
$$

which gives (i) with $C=1+3 C_{1}$.

5. The case of the unit disc. Let $D=\Delta=\{z \in C:|z|<1\}$. In [3], the proof of Lemma 1 uses Vitushkin's scheme for approximation (for example, see Chapter VIII of [2]). By adapting our argument to this special case, we obtain a much simpler proof. We restate Lemma 1 (in a slightly stronger form) for the unit disc and give a complete proof of it, which can be understood without referring to $\S 3$.

Lemma $1^{\prime}$. Let $E$ be a closed subset of $\partial \Delta$, and let $f \in H^{\infty}(\Delta)$. Assume that there is an open neighborhood $W$ of $E$ and a continuous function $u: W \rightarrow C$, such that for some $d>0$ :

$$
|f(z)-u(z)|<d \text { for } z \in \Delta \cap W .
$$

Let $S$ be any subset of $\Delta$ with $\bar{S} \cap E=\varnothing$, and let $\varepsilon>0$ be given.

We can then find $F^{\prime} \in H^{\circ}(\Delta)$ such that

(24) $F$ extends analytically across $E$ and across each arc in $\partial \Delta$ to which $f$ extends analytically.

(25) $|F(z)-f(z)|<\varepsilon$ for $z \in S$.

(26) $|F(z)-f(z)|<2 d+2 \varepsilon$ for $z \in \Delta$.

Proof. We extend $f$ to a bounded Borel function $\hat{f}$ on $C$, such that $\hat{f}=f$ on $\Delta$ and $\hat{f}$ is analytic on each arc in $\partial \Delta$ across which $f$ extends analytically. Shrinking $W$, we can assume $\bar{S} \cap W=\varnothing$. Let $\chi \epsilon$ $C_{0}^{\infty}(W)$, with $0 \leqq \chi \leqq 1$ and $\chi=1$ on a neighborhood of $E$. Define a function $h: \Delta \rightarrow C$ by 


$$
h(z)=\frac{1}{\pi} \iint_{c} \frac{\hat{f}(\zeta)-\hat{f}(z)}{\zeta-z} \frac{\partial \chi}{\partial \bar{\zeta}} d \xi d \eta .
$$

It is well known (see Gamelin [2], Chapter VIII) that $h$ has the following properties:

(a) $h \in H^{\infty}(\Delta)$, and $h$ extends analytically across each arc in $\partial \Delta$ on which $f$ is analytic.

(b) $f-h$ extends analytically across $E$.

(c) $h(z)=\chi f(z)+p(z)$ for $z \in \Delta$, where

$$
p(z)=\frac{1}{\pi} \iint_{c} \frac{\hat{f}(\zeta)}{\zeta-z} \frac{\partial \chi}{\partial \zeta} d \xi d \eta
$$

is continuous on $\bar{A}$.

Thus $h$ has similar properties as $h$ defined by (7) in $\S 3$. However, it is now trivial to approximate $h$. In fact, let $h_{r}(z)=h(r z)$ for $0<r<1$. Then $h_{r}$ is analytic on $\bar{\Delta}$. Extending $\chi u$ to be zero outside of $W$, we obtain $|\chi u(z)-\chi f(z)|<d$ for all $z \in \Delta$. Since $p$ and $\chi u$ are uniformly continuous on $\bar{\Delta}$, we can choose $r_{0}>0$ so close to 1 that

$$
\left|p(z)-p\left(r_{0} z\right)\right|<\varepsilon,\left|\chi u(z)-\chi u\left(r_{0} z\right)\right|<\varepsilon
$$

for all $z \in \bar{\Delta}$. Hence

$$
\begin{aligned}
& \quad\left|\chi f(z)-\chi f\left(r_{0} z\right)\right| \\
& \leqq|\chi f(z)-\chi u(z)|+\left|\chi u(z)-\chi u\left(r_{0} z\right)\right|+\left|\chi u\left(r_{0} z\right)-\chi f\left(r_{0} z\right)\right| \\
& <d+\varepsilon+d \text { for } z \in \Delta .
\end{aligned}
$$

Now define $F=f-h+h_{r_{0}}$. Clearly $F$ is in $H^{\circ}(\Delta), F$ extends analytically across $E$ and across each arc in $\partial \Delta$ where $f$ is analytic. It follows from (27) and (28) that

$$
|F(z)-f(z)|=\left|h(z)-h_{r_{0}}(z)\right|<2 d+2 \varepsilon \text { for } z \in \Delta,
$$

and since $S \cap W=\varnothing$, we also obtain

$$
|F(z)-f(z)|=\left|p(z)-p\left(r_{0} z\right)\right|<\varepsilon \text { for } z \in S .
$$

Lemma $1^{\prime}$ is proved.

Theorems 1, 2 and 3 follow from Lemma $1^{\prime}$ as in the general case (see §4). We can actually use the simpler inductive argument of [3], that is, we do not have to modify the domain at each step.

6. Concluding remarks. As Kerzman [6] showed, Theorem 4 holds also for strictly pseudoconvex, relatively compact domains $D$ in a Stein manifold. Hence our theorems are true in this more general situation. 
The differentiability of the boundary $\partial D$ can be relaxed. In fact, the bulk of Kerzman's proof of Theorem 4 is purely local. After a holomorphic change of coordinates in a neighborhood of $p \in \partial D$, we can assume that $\partial D$ is strictly convex (not only pseudoconvex) near $p$. As remarked by N. Øvrelid [8], this allows to reduce the order of differentiability of $\rho$ by 1 , while retaining the same estimates for the functions needed in the construction of the integration kernel. This argument shows that we only need a $C^{3}$ boundary. Actually, a $C^{2}$ boundary is sufficient, if we use some of the estimates of $\mathrm{N}$. Øvrelid [8]. He shows that the solution $u$ of $\bar{\partial} u=\alpha$ in Theorem 4 is continuous up to the boundary by approximating the integration kernel by continuous kernels. Thus the Hölder estimates in [6], which require a higher order of differentiability, are not needed.

Let $\sigma$ be Lebesgue measure on $\partial D$. It is known that every $f \in$ $H^{\infty}(D)$ has nontangential limits $\sigma$ - a.e. For a reference, see E. M. Stein [9], where a stronger statement is proved. $H^{\circ}(D)$ can thus be identified with a subalgebra of $L^{\infty}(\sigma)$. For $E \subset \partial D$, let $L_{E}^{\infty}$ be the subalgebra of $L^{\infty}(\sigma)$ of those functions which are a.e. equal to a function in $L^{\infty}$ continuous on $E$. Similarly, denote by $H_{E}^{\infty}$ the subalgebra of $H^{\circ}(D)$ of functions which extend continuously to $E$. As in [3], part of Theorem 3 can be formulated in the following way.

Theorem 5. Lst $D$ be a SSPC domain. There is a constant $C_{1}$ depending only on $D$, such that for all $E \subset \partial D$ and all $f \in H^{\infty}(D)$

$$
\operatorname{dist}\left(f, L_{E}^{\infty}\right) \leqq \operatorname{dist}\left(f, H_{E}^{\infty}\right) \leqq C_{1} \operatorname{dist}\left(f, L_{E}^{\infty}\right) \text {. }
$$

Davie, Gamelin and Garnett [1] have investigated the smallest possible constant $C_{1}$ for planar domains. It appears likely that analogous results will be valid for the domains considered here.

Since this paper was written, the author obtained the following related results. The constant $C$ in Theorem 2 can be chosen to be 1 . The constant $C_{1}$ in Theorem 5 can be chosen to be 2 . Note that the proof of Lemma 1' already gives the value 2 for closed $E \subset \partial \Delta$. The value 2 is best possible, as shown by an example of Davie, Gamelin and Garnett.

\section{REFERENCES}

1. A. M. Davie, T. W. Gamelin and J. Garnett, Distance estimates and bounded pointwise density, to appear.

2. T. W. Gamelin, Uniform Algebras, Prentice-Hall, 1969.

3. T. W. Gamelin and J. Garnett, Uniform Approximation to Bounded Analytic Functions, Revista de la Unión Matemática Argentina, 25 (1970), 87-94. 
4. H. Grauert and I. Lieb, Das Ramirezsche Integral und die Lösung der Gleichung $\bar{\partial} f=\alpha$ im Bereich der beschränkten Formen, Complex Analysis, 1969, Rice University Studies 56 (1970).

5. R. Gunning and H. Rossi, Analytic Functions of Several Complex Variables, PrenticeHall, 1965.

6. N. Kerzman, Hölder and $L^{p}$ Estimates for Solutions of $\overline{\partial u}=f$ in Strongly Pseudoconvex Domains, Comm. Pure Appl. Math., 24 (1971), 301-379.

7. I. Lieb, Ein Approximationssatz auf streng pseudokonvexen Gebieten, Math. Ann., $184(1969 / 70), 56-60$.

8. N. Øvrelid, Integral Representation Formulas and $L^{p}$ Estimates for the $\bar{\partial}$-Equation, Math. Scand., to appear.

9. E. M. Stein, Boundary values of holomorphic functions, Bull. Amer. Math. Soc., 76 (1970), 1292-1296.

Received February 3, 1971. This research is part of the author's doctoral disseration to be submitted to the University of California at Los Angeles. The author wishes to thank his advisor, Professor Theodore W. Gamelin, and Professor John Garnett for their interest in his work and for some valuable comments.

This research was partially supported by NSF Grant GP-11475.

UNiversity of California, Los ANgeles

AND

YALE UNIVERSITY 



\section{PACIFIC JOURNAL OF MATHEMATICS}

\section{EDITORS}

H. SAMELSON

Stanford University

Stanford, California 94305

C. R. Hовву

University of Washington

Seattle, Washington 98105
J. DugundJI

Department of Mathematics

University of Southern California

Los Angeles, California 90007

RICHARD ARENS

University of California

Los Angeles, California 90024

\section{ASSOCIATE EDITORS}
E. F. BECKENBACH
B. H. NeumanN
F. WOLF
K. YOSHIDA

\section{SUPPORTING INSTITUTIONS}

UNIVERSITY OF BRITISH COLUMBIA

CALIFORNIA INSTITUTE OF TECHNOLOGY

UNIVERSITY OF CALIFORNIA

MONTANA STATE UNIVERSITY

UNIVERSITY OF NEVADA

NEW MEXICO STATE UNIVERSITY

OREGON STATE UNIVERSITY

UNIVERSITY OF OREGON

OSAKA UNIVERSITY
UNIVERSITY OF SOUTHERN CALIFORNIA

STANFORD UNIVERSITY

UNIVERSITY OF TOKYO

UNIVERSITY OF UTAH

WASHINGTON STATE UNIVERSITY

UNIVERSITY OF WASHINGTON

$\stackrel{*}{*} \stackrel{*}{*} \stackrel{*}{*}{ }^{*}$ AMERICAN MATHEMATICAL SOCIETY

NAVAL WEAPONS CENTER 


\section{Pacific Journal of Mathematics}

\section{Vol. 41, No. $1 \quad$ November, 1972}

Anatole Beck and Peter Warren, Weak orthogonality.................

Jonnie Bee Bednar and Howard E. Lacey, Concerning Banach spaces whose duals are abstract $L$-spaces.............................

Louis Harvey Blake, Canonical extensions of measures and the extension of regularity of conditional probabilities..........................

R. A. Brooks, Conditional expectations associated with stochastic processes ..........................................

Theodore Allen Burton and Ronald Calvin Grimmer, On the asymptotic behavior of solutions of $x^{\prime \prime}+a(t) f(x)=e(t) \ldots \ldots \ldots \ldots \ldots \ldots$

Stephen LaVern Campbell, Operator-valued inner functions analytic on the closed disc ............................................

Yuen-Kwok Chan, A constructive study of measure theory...

Alexander Munro Davie and Bernt Karsten Oksendal, Peak interpolation sets for some algebras of analytic functions ...................

H. P. Dikshit, Absolute total-effective $\left(N, p_{n}\right)(c, 1)$ method ...............

Robert E. Edwards, Edwin Hewitt and Kenneth Allen Ross, Lacunarity for

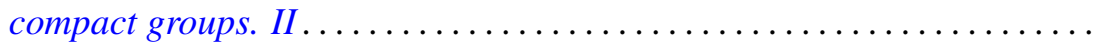

James Daniel Halpern, On a question of Tarski and a maximal theorem of Kurepa

Gerald L. Itzkowitz, A characterization of a class of uniform spaces that admit an invariant integral

Mo Tak Kiang, Semigroups with diminishing orbital diameters ..

Glenn Richard Luecke, A class of operators on Hilbert space ...

R. James Milgram, Group representations and the Adams spectral sequence. . .

G. S. Monk, On the endomorphism ring of an abelian p-group, and of a large subgroup...

Yasutoshi Nomura, Homology of a group extension ...

R. Michael Range, Approximation to bounded holomorphic functions on strictly pseudoconvex domains...

Norman R. Reilly, Inverse semigroups of partial transformations and $\theta$-classes.

Chris Rorres, Strong concentration of the spectra of self-adjoint operators

Saharon Shelah, A combinatorial problem; stability and order for models and theories in infinitary languages.

George Gustave Weill, Vector space decompositions and the abstract

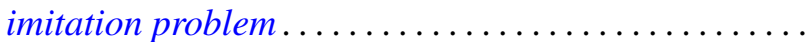

